\title{
PROPOSTA DE AUTOMAÇÃO DE UMA SUBESTAÇÃO DE 13.8KV PARA O ALCANCE DE EFICIÊNCIA ENERGÉTICA DE UM HOSPITAL EM TERESINA ${ }^{1}$
}

\author{
Lucas Rocha de Abrantes Duarte ${ }^{2}$ - Centro Universitário Santo Agostinho \\ Gabriel Messias Mamede Costa ${ }^{3}$ - Centro Universitário Santo Agostinho \\ Marcielly dos Reis Macêdo ${ }^{4}$ - Centro Universitário Santo Agostinho \\ Shélyda Raiane Rodrigues Machado ${ }^{5}$ - Centro Universitário Santo Agostinho \\ Brenda Virna Sousa Rocha ${ }^{6}$ - Centro Universitário Santo Agostinho \\ Thiago Pereira de Sousa ${ }^{7}$ - Centro Universitário Santo Agostinho \\ Prof. Me. Vinícius Magno Uchôa Lima Oliveira ${ }^{8}$ - Centro Universitário Santo Agostinho
}

\section{RESUMO}

Dentro do ambiente hospitalar, a energia elétrica tem um papel essencial, desde manter as luzes acesas, garantir o funcionamento dos equipamentos de suporte à vida bem como assegurar as atividades e os processos existentes. Neste contexto, monitorar e controlar a qualidade da energia elétrica é importante para que o hospital desempenhe seu objetivo principal: garantir o atendimento de qualidade e a vida de pacientes ali alocados. $\mathrm{O}$ controle e automação, um dos ramos da engenharia elétrica, surge como potencial solução para essa necessidade. Portanto, o principal objetivo deste artigo é a proposta de automatização do sistema de monitoramento, atualmente manual, em busca da eficiência energética de uma subestação de $13.8 \mathrm{KV}$ em um hospital em Teresina. Essa automatização será possível através da coleta e controle dos parâmetros elétricos de uma subestação hospitalar, tais como tensão, corrente, frequência e temperatura, contando também com a interação de um Controlador Lógico Programável (CLP) e do Controle de Supervisão e Aquisição de Dados (SCADA). Este último é definido como um sistema centralizado que controla e monitora todos os parâmetros da subestação e garante que os dados principais dos equipamentos sejam analisados através de um monitor de um computador. Desse modo, o modelo será projetado para o controle e análise de parâmetros dos equipamentos em tempo real, promovendo um monitoramento seguro, confiável e eficiente, capaz de otimizar a qualidade de energia e segurança da subestação e do hospital.

\footnotetext{
${ }^{1}$ Trabalho apresentado no Congresso Brasileiro Ciência e Sociedade (CBCS 2019), promovido pelo Centro Universitário Santo Agostinho, de 03 a 05 de outubro de 2019, em Teresina-PI.

${ }^{2}$ Acadêmico do $10^{\circ}$ período do curso de Bacharelado em Engenharia Elétrica - UNIFSA, lucasrocha4034@gmail.com.

${ }^{3}$ Acadêmico do $10^{\circ}$ período do curso de Bacharelado em Engenharia Elétrica - UNIFSA, messiasgabriel@hotmail.com.

${ }^{4}$ Acadêmica do $10^{\circ}$ período do curso de Bacharelado em Engenharia Elétrica - UNIFSA, marciellymary@hotmail.com.

${ }^{5}$ Acadêmica do $10^{\circ}$ período do curso de Bacharelado em Engenharia Elétrica - UNIFSA, shelydarodrigues@gmail.com.

${ }^{6}$ Acadêmica do $10^{\circ}$ período do curso de Bacharelado em Engenharia Elétrica - UNIFSA, brendavirna@hotmail.com.

${ }^{7}$ Acadêmico do $10^{\circ}$ período do curso de Bacharelado em Engenharia Elétrica - UNIFSA, tp.sousa1909@hotmail.com.

${ }^{8}$ Docente do curso de Bacharelado em Engenharia Elétrica - UNIFSA, viniciusuchoa@gmail.com.
} 
ANAIS CBCS 2019 | 3 a 5 de outubro de 2019 | Centro Universitário Santo Agostinho - Teresina - PI

Palavras-Chave: Subestação; Automação; Eficiência energética.

\section{ABSTRACT}

Within the hospital environment, electricity plays an essential role, from keeping the lights on, ensuring the functioning of life support equipment, as well as ensuring existing activities and processes. In this context, monitoring and controlling the quality of electricity is important for the hospital to fulfill its main objective: to ensure quality care and the lives of patients allocated there. Control and automation, one of the branches of electrical engineering, emerges as a potential solution to this need. Therefore, the main objective of this paper is the proposal of automation of the monitoring system, currently manual, in search of the energy efficiency of a 13.8 KV substation in a hospital in Teresina. This automation will be possible through the collection and control of electrical parameters of a hospital substation, such as voltage, current, frequency and temperature, as well as the interaction of a Programmable Logic Controller (PLC) and the Supervisory Control and Data Acquisition ( SCADA). The latter is defined as a centralized system that controls and monitors all substation parameters and ensures that main equipment data is analyzed through a computer monitor. Thus, the model will be designed for the control and analysis of equipment parameters in real time, promoting safe, reliable and efficient monitoring capable of optimizing substation and hospital power quality and safety.

Keywords: Substation; Automation; Energy efficiency.

\section{INTRODUÇÃO}

O suprimento de energia elétrica é um dos sistemas mais complexos existentes. Desse modo, a eficiência energética e operação desse sistema, torna-se a principal preocupação. A automação em um sistema elétrico de potência, visa controlar automaticamente o sistema de energia através dos dispositivos de instrumentação de controle [16].

A automação de subestações de energia elétrica, está relacionada a coleta de dados de dispositivos eletrônicos inteligentes (IED), controle e automação, instalações de componentes de monitoramento dentro da subestação, bem como o uso de sistemas de controle para a obtenção dos dados remotamente do sistema automatizado [5].

Um método tradicional de monitoramento em uma subestação hospitalar pode proporcionar falhas e distúrbios graves no âmbito geral, promovendo o risco de centenas de pessoas que dependem do suporte de equipamentos ou cirurgias nesse ambiente. 


\section{CONGEESSOC CIENCIAESOCIEDADE

ANAIS CBCS 2019 | 3 a 5 de outubro de 2019 | Centro Universitário Santo Agostinho - Teresina - P|

Nesse contexto, faz-se necessário implantar um sistema de monitoramento que seja capaz de detectar, monitorar e classificar remotamente os principais dados dos equipamentos do ambiente de estudo, a fim de otimizar a qualidade de energia, manter o fornecimento contínuo e aumentar a segurança local.

O principal objetivo do artigo é a proposta de automatização de uma subestação (SE) de 13,8kV do hospital localizado na cidade de Teresina para análise e controle dos principais dados dos equipamentos da SE, a fim de garantir a eficiência energética do hospital, bem como a otimização do monitoramento atual. Este sistema de controle utiliza como ferramenta o Controle de Supervisão e Aquisição de Dados (SCADA), o qual é utilizado para monitorar e controlar uma planta ou equipamento em diversos setores como telecomunicações [2], controle de água e resíduos, sistema de refrigeração, petróleo, gás e transporte.

Os dados de campo, são encaminhados para a estação de controle através de um dispositivo de interface de campo utilizando protocolos de comunicação. A fim de garantir a transferência dos dados, são utilizados cabos de comunicação ou outros meios como a comunicação via satélite ou em rede [4].

A estação de controle coleta os sinais recebidos, interpreta e processa os dados, em seguida uma solução é criada e encaminhada. Por fim, a subestação retransmite esta solução de volta ao dispositivo de interface de campo com um formato compatível para o reconhecimento pelo dispositivo [13].

Para alcançar este objetivo é necessária a realização de algumas etapas, tais como:

- Levantamento histórico dos principais blocos do hospital que possuem um maior consumo a fim de melhorar a qualidade de energia;

- Verificar os principais equipamentos da subestação para implementação do monitoramento e controle;

- Analisar os dados coletados através do monitoramento e do controle, remotamente ao longo do período; 
ANAIS CBCS 2019 | 3 a 5 de outubro de 2019 | Centro Universitário Santo Agostinho - Teresina - P

- Mensurar as soluções mais viáveis para a eficiência energética do hospital e otimização da subestação a fim de conter os problemas de maior frequência;

Com o crescimento significativo da energia elétrica no cenário mundial, juntamente com o aumento da tecnologia da informação, a demanda por meios de comunicação e monitoramento em tempo real nas redes de distribuição de energia elétrica tem aumentado gradativamente em busca de elevar a eficiência energética tanto para os consumidores como para a concessionária de energia elétrica. Diante disso, este projeto tem como proposta implementar um sistema de controle e monitoramento automático em uma subestação de $13.8 \mathrm{KV}$ de um hospital em Teresina, por meio de um Controlador Lógico Programável (CLP) e do Controle de Supervisão e Aquisição de Dados (SCADA), com a finalidade de dispor melhorias do sistema atual, tornando o processo mais eficiente e econômico.

\section{METODOLOGIA}

Inicialmente o estudo propõe pesquisas em artigos científicos e periódicos, analisando os principais distúrbios que ocorrem em subestações de energia elétrica, bem como suas características predominantes, causas e consequências mais frequentes para o ambiente de estudo. Em seguida, o projeto terá continuidade com o monitoramento, análise e coleta de dados de corrente, tensão e temperatura dos equipamentos locados na subestação de $13.8 \mathrm{KV}$. Em seguida, com a obtenção destes parâmetros, serão executadas as principais medidas em busca da eficiência energética local, bem como a automatização desta subestação, realizando a coleta de dados em tempo real e enviando estes para um servidor.

Nas pesquisas bibliográficas serão abordados dados e informações de periódicos e artigos científicos que tratam acerca da automatização, CLP, subestação hospitalar, entre outros. Na análise e coleta de dados serão incluídas informações dos 


\section{cONGEESSOC CIENCIAESOCIEDADE

ANAIS CBCS 2019 | 3 a 5 de outubro de 2019 | Centro Universitário Santo Agostinho - Teresina - P|

equipamentos que serão monitorados na subestação e principais setores e blocos do hospital.

Em seguida será implementado um circuito monitorador que irá coletar os dados como: níveis de tensão, corrente em todas as fases e temperatura. Esses dados serão enviados por meio de comunicação sem fio para um servidor localizado na sala de engenharia do hospital, facilitando assim a tomada de decisão quanto ao suprimento de energia e parâmetros da rede. O circuito proposto será abordado ao decorrer do projeto, o mesmo terá como componente principal um CLP e os respectivos sensores para coleta dos parâmetros da rede.

Em suma, o projeto proposto é de fundamental importância tanto para a subestação de $13.8 \mathrm{kV}$ quanto para o hospital. Ao longo do desenvolvimento da pesquisa, será possível obter informações dos principais distúrbios e variações de tensão, corrente e temperatura em tempo real, além da frequência com que estes fenômenos ocorreram. A partir desenvolvimento do projeto proposto, o presente artigo contribuirá com novas soluções de eficiência energética e para a otimização do monitoramento e controle da subestação no hospital.

\section{RESULTADOS E DISCUSSÃO}

Em redes hospitalares, áreas como bloco cirúrgico, salas de emergência e laboratórios de análise são supridos pela rede de emergência, ou seja, são alimentados por duas fontes de energia elétrica distintas, conhecido como unidades de redes ininterruptas (UPS). Esses sistemas são implantados em áreas que demandam uma energia elétrica de alta qualidade e confiança. Em redes elétricas de hospitais, há a necessidade de uma maior segurança quando se trata do monitoramento da subestação de energia, as instalações e os equipamentos devem estar sujeitos a operar em situações de emergência, como faltas ou desligamentos ocasionados internamente. Distúrbios como esses, caso não sejam supridos ou monitorados de imediato, podem promover riscos para centenas de pacientes. 
ANAIS CBCS 2019 | 3 a 5 de outubro de 2019 | Centro Universitário Santo Agostinho - Teresina - P

Atualmente, as redes de subestações ainda sofrem com interrupções de energia e blackouts devido a carência de análises automatizadas e visibilidade da rede elétrica através das concessionárias. Com o crescimento das redes de distribuição e sua complexidade [6], o avanço da tecnologia da informação e a chegada da indústria 4.0, a real necessidade da automatização de subestações de energia para o aumento da eficiência e melhoria da qualidade de energia é agora mais evidente.

Sistemas de controle e monitoramento remoto usando CLP e SCADA podem promover um aprimoramento da qualidade de energia e no monitoramento mais eficiente, em segundos, sem que haja necessidade de um operador se deslocar com frequência para diagnosticar a causa do problema. Por isso, faz-se necessário aderir a um sistema que seja capaz de monitorar, detectar e classificar automaticamente a presença de distúrbios ocorridos na subestação.

Um controlador Lógico Programável (PLC), é um computador industrial de sistemas de controle, que monitora automaticamente o estado de entrada dos dispositivos e toma decisões com base em uma programação personalizada a fim de controlar o estado dos dispositivos de saída. Um dos controladores utilizados para automação de subestações de energia elétrica é o PLC de Allen Bradley, pois este modelo possui processadores de fácil acesso e procedimentos simples para o controle e monitoramento da subestação [6]. Dessa forma, estas aplicações são bastantes difundidas com o uso dos sistemas PLC e SCADA, por conta de seus processadores de vastas aplicações como no manuseio de materiais, operações de montagem, processos de monitoramento e dentre outros. Dentre as várias linguagens de programação utilizadas para operar o PLC, a mais utilizada atualmente é ladder Logic (LAD), esta é considerada como a melhor linguagem compatível para automação de subestação com o número mínimo de comandos necessários e maior precisão.

O sistema SCADA, basicamente é um tipo de aplicativo onde se deve obter a interface entre homem e máquina, com o operador estando presente na sala de controle analisando os dados. O aplicativo WonderwarelnTouch é considerado com um 


\section{conyegESSO CIENCIAESOCIEDADE

ANAIS CBCS 2019 | 3 a 5 de outubro de 2019 | Centro Universitário Santo Agostinho - Teresina - P|

dos softwares adequados para executar a operação no SCADA [14]. Com esse aplicativo, cerca de $3 \%$ da taxa de sucesso no tratamento das execuções são alcançadas, e $41 \%$ do tempo total das tarefas completas podem ser reduzidas. Além disso, este garante a redução de custos de capital e operações, melhoria operacional, eficiência e redução de interrupções.

A automação é o processo de controlar e monitorar automaticamente dados e parâmetros de um processo, com auxílio dos dispositivos de automação existentes. Inicialmente, antes da automação, o controle de processos nas usinas era supervisionado manualmente com base em instrumentos instalados em campos. Isso exigia que o supervisor local, estivesse presente a todo momento no local.

Com o desenvolvimento de dispositivos eletrônicos inteligentes, houve um grande salto para o aprimoramento de subestações de energia. Uma vasta quantidade de informações é gravada frequentemente ou capturada quando o dispositivo é acionado por um evento decorrente de uma falta ou algum distúrbio na rede elétrica [2]. Critérios para o sistema de monitoramento proposto neste projeto são levados em consideração como: custo, segurança de rede e confiabilidade, com o intuito de garantir que o monitoramento a ser implantado na subestação hospitalar de fato alcance a eficiência.

\section{CONSIDERAÇÕES FINAIS}

Com o desenvolvimento do projeto, espera-se abordar uma nova visão no que diz respeito ao monitoramento e automação de subestações de energia elétrica no âmbito hospitalar com intuito de obter a eficiência energética do local. Tais monitoramentos, quando realizados remotamente em tempo real, podem obter melhorias no sistema quando comparados aos métodos tradicionais realizados. Promovendo a qualidade de energia a ser fornecida para o hospital, bem como a segurança da subestação. Em suma, com a elaboração do projeto proposto, espera-se que o monitoramento possa ser realizado remotamente, através do sistema CLP e 


\section{congersSOCOENCIAESOCIEDADE}

ANAIS CBCS 2019 | 3 a 5 de outubro de 2019 | Centro Universitário Santo Agostinho - Teresina - P

SCADA, obtendo dados e informações em tempo real, a fim de otimizar o modelo atual existente.

\section{REFERÊNCIAS}

[1] Amit Sachan, "Microcontroller based substation monitoring and control system with GSM modem" ISSN: 2278-1676 Volume 1, Issue 6 (July-Aug. 2012), PP 13-21.

[2] A. Bruce, "Reliability analysis of electric utility scada systems," Power Systems, IEEE Transactions on, vol. 13, no. 3, pp. 844-849, Aug 1998.

[3] M. S. Thomas, P. Kumar, and V. K. Chandna, "Design, development, and commissioning of a supervisory control and data acquisition (scada) laboratory for research and training," Power Systems, IEEE Transactions on, vol. 19, no. 3, pp. 15821588, 2004.

[4] Argonne National Laboratory, "Assessment of the Potential Costs and Energy Impacts of Spill Prevention, Control, and Countermeasure equirements for Electric Utility Substations", Draft Energy Impact Issue Paper, 2006.

[5] B. A. Carreras, V. E. Lynch, D. E. Newman and I. Dobson, "Blackout Mitigation Assessment in Power Transmission Systems", Hawaii International Conference on System Science, January 2003. 
ANAIS CBCS 2019 | 3 a 5 de outubro de 2019 | Centro Universitário Santo Agostinho - Teresina - P

[6] Allen-Bradley SLC 500 Modular Hardware style User manual from Rockwell Automation.

[7] N. Arghira, D. Hossu, I. Fagarasan, S. S. Iliescu, and D. R. Costianu, "Modern scada philosophy in power system operation-a survey," University" Politehnica" of Bucharest Scientific Bulletin, Series C: Electrical Engineering, vol. 73, no. 2, pp. 153-166, 2011.

[8] Daponte, M. Di Penta and G. Mercurio, "TRANSIENTMETER: A Distributed Measurement System for Power Quality Monitoring", IEEE Transactions on Power Delivery, Vol. 19, Issue. 2, pp: 456-463, 2004.

[9] I. Fagarasan, S. S. Iliescu, and N. Arghira, "Advances in power system control," in Proceedings of the 1st Workshop on Energy, Transport and Environmental Control Applications, 2009, pp. 62-71.

[10] FERNANDES, L.M. A energia elétrica nas instalações hospitalares. Disponível em: < https://www.voltimum.pt/artigos/artigos-tecnicos/energia-electrica-nas $>$. Acesso em: 12 fev. 2019.

[11] G. Pudlo, S. Tenbohlen, M. Linders and G. Krost, "Integration of Power Transformer Monitoring and Overload Calculation into the Power System Control Surface", IEEE/PES Transmission and Distribution Conference and Exhibition, Vol. 1, pp: 470-474 Asia Pacific, 2002.

[12] Jyotishman Pathak, Yuan Li, Vasant Honavar and James D. McCalley, "A ServiceOriented Architecture for Electric Power Transmission System Asset Management", In ICSOC Workshops, pp: 26-37, 2006.

[13] M. Kezunovic, Y. Guan, M.Ghavami, "New concept and solution for monitoring and control system for the 21 st century substation" (IEEE).

[14] Natalie Matta, Rana Rahim-Amoud, Leila Merghem-Boulahia, Akil Jrad, "A wireless sensor network for substation monitoring and control in the smart grid" (IEEE)

[15] Phutane, Pravin S., and A. K. Jhala. "INTERNATIONAL JOURNAL OF ELECTRICAL ENGINEERING \& TECHNOLOGY (IJEET)." Journal Impact Factor 5.5 (2014): 36-43.

[16] Phutane, Pravin S., et al. "Graphene photovoltaic panel (GPP)." Advances in Electrical, Electronics, Information, Communication and Bio-Informatics (AEEICB), 2017 Third International Conference on. IEEE, 2017. 


\section{congersSOCOENCIAESOCIEDADE}

ANAIS CBCS 2019 | 3 a 5 de outubro de 2019 | Centro Universitário Santo Agostinho - Teresina - P

[17] R.R. Negenborn, A.G. Beccuti, T. Demiray, S. Leirens, G. Damm, B. De Schutter and M. Morari, "Supervisory hybrid model predictive control for voltage stability of power networks", Proceedings of the 2007 American Control Conference, New York, New York, pp: 5444-5449, July 2007.

[18] R. B. Roy, "Controlling of electrical power system network by using scada," International Journal of Scientific \& Engineering Research, vol. 3, no. 10, Oct 2012.

[19] WonderwareFactory Suite In Touch Users Guide.

[20] Xiaomeng Li and Ganesh K. Venayagamoorthy, "A Neural Network Based Wide Area Monitor for a Power System", IEEE Power Engineering Society General Meeting, Vol. 2, pp: 1455-1460, 2005.

[21] Zhi-Hua Zhou, Yuan Jiang, Xu-Ri Yin, and Shi-Fu Chen, "The Application of Visualization and Neural Network Techniques in a Power Transformer Condition Monitoring System", In: T. Hendtlass and M. Ali eds. Lecture Notes in Artificial Intelligence 2358, Berlin: Springer- Verlag, pp: 325-334, 2002.

[22] DHIMAR, M. K. Substation Monitoring and Control Using Microcontroller \& GSM. v. 04, n. 04, p. 6, [s.d.].

[23] M. S. Thomas, P. Kumar, and V. K. Chandna, "Design, development, and commissioning of a supervisory control and data acquisition (scada) laboratory for research and training," Power Systems, IEEE Transactions on, vol. 19, no. 3, pp. 15821588, 2004. 